\title{
Complexity Inside and Outside the Brain: How to Manage Internal (Interoceptive) and External (Domotics) Environment during Adaptive Inter- Actions ${ }^{+}$
}

\author{
Laura Angioletti and Michela Balconi \\ Research Unit in Affective and Social Neuroscience, Department of Psychology, Catholic University of the \\ Sacred Heart, Milan, Italy \\ + Presented at the Entropy 2021: The Scientific Tool of the 21st Century, 5-7 May 2021; Available online: \\ https://sciforum.net/conference/Entropy2021/.
}

Published: 5 May 2021

Previous studies supported the notion that brain activity is slightly sub-critical in normal waking consciousness (Priesemann et al., 2013), and in this way, it can exert better control over the rest of the world, most of which is critical. This control may take the form of managing endogenous processes within the brain or interacting with the environment in order to functionally shape it (Carhart-Harris et al., 2014). The relationship between complex systems, i.e., human-to-environment relation, from an adaptive perspective is mediated by the sensory system with the main goal of maintaining a balance, aiming for harmony and avoiding ruptures. Through the description of two pilot studies, the advantages of adopting neuroscientific tools for exploring neurophysiological brain-and-body activity during complex inter-actions will be elucidated. This research has been conducted in two different fields of application: the first one investigated how the person answer to a complex domotic environment; the second one regards the deepening of interoceptive awareness as a possible factor influencing functional empathic response to external challenge. In the first study, some distinguishing effects of domotics on users' cognitive and emotional behavior are highlighted by using the neuroscientific approach. In order to define effects of Smart Home System (SHS) on UX, a neuroscientific wireless multi-methodology was adopted with the purpose of recording and confronting the neural activity (Electroencephalography, EEG) and autonomic system responses (with Biofeedback) of 19 individuals during a resting state (RS) baseline and the exploration of 5 different tech-interaction areas in a domotic environment. In the second study, a BIO-EEG-fNIRS (functional Near Infrared Spectroscopy) co-registration approach was adopted while 20 healthy participants performed a new paradigm for investigating the effects of interoceptive ability on empathy. Overall, the advantages and limitations of the applications of neuroscientific paradigms and tools for analyzing human interaction with complex systems will be discussed.

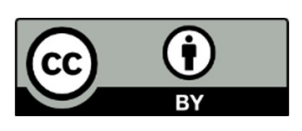

(C) 2021 by the authors. Licensee MDPI, Basel, Switzerland. This article is an open access article distributed under the terms and conditions of the Creative Commons Attribution (CC BY)

license (http://creativecommons.org/licenses/by/4.0/). 